\title{
Diagnose, understand, embrace
}

\author{
The Autistic Brain: Thinking Across \\ the Spectrum \\ Temple Grandin and Richard Panek \\ Houghton Mifflin Harcourt; 2013
}

$\mathrm{P}$ hysicians know that human experience is infinitely complex and variable. The insight that "everyone is unique" sounds like a T-shirt slogan or a platitude that a therapist might recite reassuringly, but doctors have both a scientific and clinical perspective that affirm the statement's profound truth. Every person is different, inside and out. Our preferences, sensitivities, strengths, emotions and cognitive processes combine in ways that can never be replicated, even if the effects of varying environments and experiences could somehow be factored out of the equation.

At the same time, there are ways in which we are more or less similar. The tension between sameness and difference may lead us to categorize people. This can be helpful when we identify something about group members that provides insight into what makes them the way they are. But, categorizing people can be harmful if it shuts down our curiosity, blinds us to within-group differences or leads to assumptions about the capabilities of members of the group. When categorization is part of making a medical diagnosis, the implications can be profound for the individuals and for society.

The problem of how to diagnose, understand and embrace conditions on the autism spectrum is the subject of The Autistic Brain: Thinking Across the Spectrum by Temple Grandin and Richard Panek. Grandin is a well-known animal scientist who has autism. She has written a number of commercially successful books about autism and animal welfare intended for a general audience and is the subject of a biographical film. Her collaborator, Richard Panek, is a science jour-

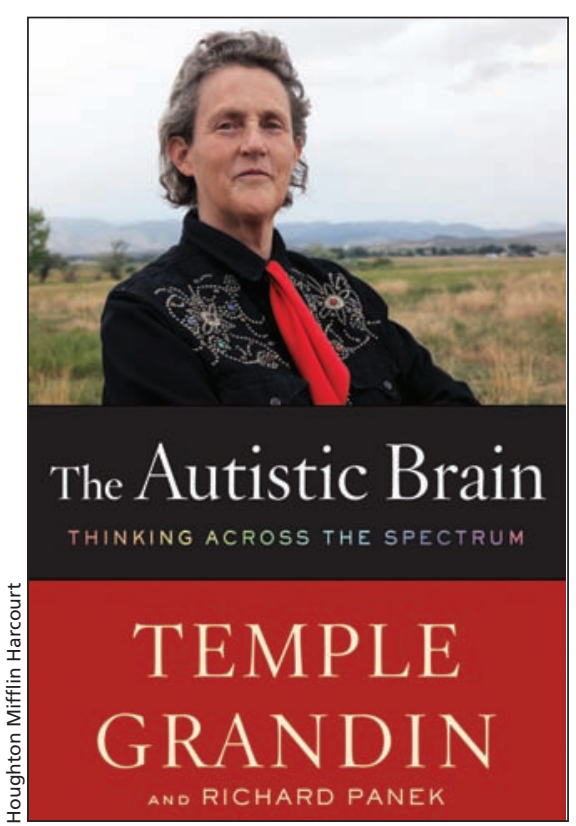

nalist. In The Autistic Brain, Grandin and Panek draw heavily on Grandin's experiences as a person with autism to provide examples and insight relating to the advances in biological and neurocognitive understanding of the condition.

The book is divided into two sections. The first, titled "The autistic brain," opens with a historic overview of how autism has been diagnosed and some of the early theories about etiology. It then reviews current neuroimaging findings about the brains of people with autism spectrum disorders, as well as efforts to identify underlying genetic factors. Grandin uses her own neuro imaging results as a case study as she discusses how the heterogeneous findings in the literature can apply to individuals. She clearly finds it helpful to relate her neuroanatomy to her experiences; for example, learning she has enlarged amygdalae helped her make sense of the panic attacks she experienced in the 1970s. In the second section of the book, "Rethinking the autistic brain," Grandin and Panek explore what is known about the cognitive and sensory aspects of autism, especially the importance of visual thinking and recognition of patterns.

Even though The Autistic Brain aims to be scientific and objective, it is still a very personal book. It is not possible to separate Grandin's narrative from the subject matter, even though this is not, strictly speaking, an autobiography or memoir. She talks about her personal and professional life throughout, but in an incomplete way that leaves many unanswered questions. It is possible that these gaps could be filled in by reading Grandin's other books or by watching the film about her life. But for the reader encountering her for the first time, it is not always easy to piece the story together. Even more striking is the impersonal way Grandin approaches the personal. She repeatedly reports on her own cognitive processes and viewpoints in a detached, observational manner. One could argue that it would be difficult to do otherwise and still be analytical about her experiences, but the other possibility is that this detachment is a reflection of Grandin's cognitive processes. This will interest some readers, but may be off-putting for others.

This book will likely interest physicians whose practices focus on autism spectrum disorders. It may also be appropriate for people with autism spectrum disorders and their families, because it includes suggestions on how to capitalize on an individual's strengths. In general, the authors are optimistic that increasing scientific knowledge about autism, combined with a more nuanced understanding of the cognitive and sensory processes, will lead to more accurate diagnoses and better quality of life for people with autism spectrum disorders.

\section{Lara Hazelton MD}

Psychiatrist

Halifax, NS

CMAJ 2014. DOI:10.1503/cmaj.140042 